\title{
Electric Quadrupolar Contribution to the Nuclear Spin-Lattice Relaxation of Ir in Fe
}

\author{
G. Seewald, E. Zech, and H.J. Körner \\ Physik-Department, Technische Universität München, D-85748 Garching, Germany \\ D. Borgmann \\ Institut für Physikalische und Theoretische Chemie, Universität Erlangen-Nürnberg, D-91058 Erlangen, Germany \\ M. Dietrich \\ Technische Physik, Universität des Saarlandes, D-66041 Saarbrücken, Germany
}

ISOLDE Collaboration

CERN, CH-1211 Geneva 23, Switzerland

(Received 13 July 2001; published 16 January 2002)

\begin{abstract}
We report on the first quantitative determination of the electric quadrupolar contribution to the nuclear spin-lattice relaxation in a transition metal. For ${ }^{186} \mathrm{Ir}$ and ${ }^{189} \mathrm{Ir}$ in Fe we have determined the magnetic and the electric quadrupolar part of the relaxation for magnetic fields between 0.01 and $2 \mathrm{~T}$. The quadrupolar part gives information on the role of the orbital motion of the electrons for the relaxation process. Our results prove that the unexpected high relaxation rates in $\mathrm{Fe}$ and their magnetic field dependence are due to a nonorbital relaxation mechanism.
\end{abstract}

DOI: 10.1103/PhysRevLett.88.057601

PACS numbers: 76.60.Es, 75.50.Bb, 76.80.+y

The nuclear spin-lattice relaxation in metals arises predominantly from the magnetic hyperfine interaction between the nuclear spins and the conduction electrons. It gives information on the density of states at the Fermi energy and the magnitude of low frequency spin fluctuations [1-3].

The interpretation of the relaxation is complicated in transition metals by the fact that several relaxation mechanisms can contribute, but only the total relaxation rate $R$ is usually measured: According to the type of the responsible hyperfine interaction, $R$ can be subdivided into an orbital $\left(R_{o}\right)$, a Fermi-contact $\left(R_{c}\right)$, a spin-dipolar $\left(R_{s d}\right)$, a core-polarization $\left(R_{c p}\right)$, and a quadrupolar $\left(R_{q}\right)$ contribution $[3,4]$. The first four contributions represent together the magnetic relaxation rate $R_{m} . R_{q}$ is due to the electric hyperfine interaction and arises from electric field gradient fluctuations at the nuclear site.

The various contributions involve quite different electronic excitations: $R_{c}$, for example, is connected to spin flips of $s$ electrons; $R_{o}$ and $R_{q}$ are connected to orbital momentum changes of $p$ or $d$ electrons. A separate determination would, therefore, give valuable information on the relaxation process. Such information is especially desirable since experiment and theory are in serious disagreement for several transition metal systems $[5,6]$.

In this situation, one can make use of two special features of $R_{q}: R_{q}$ can be determined separately and it is so closely related to $R_{o}$ that $R_{o}$ can be estimated from $R_{q}$ $[4,7]$. This offers the unique possibility to decompose experimentally the relaxation into an orbital and a nonorbital part.

This possibility is used here for the first time. It is used to study the origin of two still unsolved problems in the nuclear spin-lattice relaxation in Fe: (i) The relaxation rates are usually larger than predicted by $a b$ initio calculations. This effect is especially prominent and well documented for the $5 d$ impurities in Fe [6]. (ii) For magnetic fields below $1 \mathrm{~T}$ there is a so far unexplained magnetic field dependence due to which the relaxation in zero field is typically 3 to 10 times faster than at high fields [8-10].

This work is also the first experimental determination of $R_{q}$ in a transition metal, although the presence of this contribution to the relaxation in transition metals is meanwhile well established in the theoretical work [4,7,11-13]. In Ref. [14] the relaxation of ${ }^{97} \mathrm{Mo}$ in Mo was interpreted by the presence of a quadrupolar contribution. But the quadrupole moment of ${ }^{97} \mathrm{Mo}$ [15], which was not known at that time, does not support this interpretation. A quadrupolar contribution to the relaxation was also reported for ${ }^{103} \mathrm{Ru}$ in $\mathrm{Ru}[11,16]$. But no quantitative conclusions are possible since the relaxation data on ${ }^{103} \mathrm{Ru}$ were interpreted in terms of the "high temperature limit" which is not justified for that experiment.

$R_{m}$ and $R_{q}$ can be separated by using the scaling of $R_{m}$ and $R_{q}$ with the square of the magnetic and the quadrupole moment, respectively [4]:

$$
\begin{gathered}
R=\left(R_{m} / g^{2}\right) g^{2}+\left(R_{q} / N_{q}\right) N_{q}, \\
g=\frac{\mu}{I}, \quad N_{q}=\frac{Q^{2}(2 I+3)}{I^{2}(2 I-1)} .
\end{gathered}
$$

$I, \mu, g$, and $Q$ are the spin, the magnetic moment, the $g$ factor, and the quadrupole moment of the respective isotopes. We assume that $\mu$ is given in nuclear magnetons and $Q$ in barns. $g$ and $N_{q}$ are then dimensionless quantities. $R_{m} / g^{2}$ and $R_{q} / N_{q}$ are isotope independent measures 
of the magnetic and the quadrupolar part of the relaxation. They can be determined from the relaxation rates of two isotopes of the same element with different nuclear moments using Eq. (1). However, only for a few isotopes with large quadrupole moment and small magnetic moment is there a measurable quadrupolar contribution at all. For most isotopes, $R_{q}$ can be neglected with respect to $R_{m}$ [7].

We have chosen radioactive ${ }^{189} \operatorname{Ir}\left(I^{\pi}=3 / 2^{+}\right)$and ${ }^{186} \operatorname{Ir}$ $\left(I^{\pi}=5^{+}\right)$as the most suitable isotope pair for our problem. One of the largest $(Q / \mu)$ ratios among all available $5 d$ isotopes leads to a particularly large electric quadrupolar contribution to the relaxation of ${ }^{189} \mathrm{Ir}$. On the other hand, the very convenient decay properties of ${ }^{186} \mathrm{Ir}$ allow particularly precise relaxation measurements by nuclear magnetic resonance on oriented nuclei (NMR-ON). The relaxation measurements on the two isotopes were performed in the same sample in the same experiment with the same technique. They are thus directly comparable.

An Fe single crystal disk, $2.2 \mathrm{~mm}$ thick and $12 \mathrm{~mm}$ in diameter, was used as host. The disk plane was perpendicular to the [110] direction. Since the probe nuclei were implanted into the first $50 \mathrm{~nm}$, much attention was paid to the surface preparation. The final cleaning of the surface was performed by a sequence of $\mathrm{Ar}^{+}$ion sputtering and annealing steps.

${ }^{186} \mathrm{Hg}$ and ${ }^{189} \mathrm{Hg}$, the precursors of ${ }^{186} \mathrm{Ir}$ and ${ }^{189} \mathrm{Ir}$, were implanted at the on-line mass separator ISOLDE at CERN. The $\mathrm{Hg}$ isotopes were produced in spallation reactions using the $1 \mathrm{GeV}$ CERN PS-booster proton beam and the liquid $\mathrm{Pb}$ target. The implantation voltage was $60 \mathrm{kV}$, and the implantation dose was about $0.6 \times 10^{14} \mathrm{~cm}^{-2}$ and $1.6 \times 10^{14} \mathrm{~cm}^{-2}$ for $A=186$ and $A=189$, respectively. After the implantation the sample was annealed for $1 / 2 \mathrm{~h}$ at $970 \mathrm{~K}$, before it was mounted into a ${ }^{3} \mathrm{He}-{ }^{4} \mathrm{He}$ dilution refrigerator and cooled down to temperatures in the $10 \mathrm{mK}$ range. The external magnetic field $B_{\text {ext }}$ was applied in the disk plane parallel to the [100] direction.

For each isotope first the magnetic and electric hyperfine splitting frequencies were measured by NMR-ON and modulated adiabatic fast passage on oriented nuclei. They agreed well with the known hyperfine field and the electric field gradient of Ir in Fe [17], and the known magnetic and quadrupole moments of ${ }^{186} \operatorname{Ir}$ and ${ }^{189} \operatorname{Ir}$ [18].

In relaxation measurements by the NMR-ON technique [19] the anisotropy of the $\gamma$ radiation in the decay of oriented probe nuclei is used to monitor the time dependence of the nuclear orientation. The rf field at the NMR resonance frequency is applied first without frequency modulation (FM). Virtually no nuclear spins are excited because of the large inhomogeneous broadening of the hyperfine splitting, and the nuclear spins are in thermal equilibrium. At the time $t_{\text {on }}$ the FM is switched on and the nuclear orientation is partly destroyed. After some time a new equilibrium is reached. At the time $t_{\text {off }}$ the FM is switched off and the nuclear orientation relaxes back to the thermal equilibrium value.
Typical NMR-ON time spectra for ${ }^{186}$ Ir and ${ }^{189}$ Ir are shown in Fig. 1. The relaxation constants and the rf field strengths were determined from these time spectra via least squares fit. To describe the time dependence of the $\gamma$ anisotropy the rate equations for the population numbers of the sublevels were solved [19]. These rate equations were extended by the rf transition probabilities [9] and by the transition rates of the quadrupolar part of the relaxation [4].

The magnetic and electric quadrupolar relaxation rates, $R_{m}$ and $R_{q}$, are conveniently given as inverse Korringa constants, so that the Korringa relation is given by $\left(T_{1} T\right)^{-1}=$ $\left(R_{m}+R_{q}\right)$. The ratios $R_{m}^{(186)} / R_{m}^{(189)}$ and $R_{q}^{(186)} / R_{q}^{(189)}$ are known from the scaling of the relaxation rates with the nuclear moments. With this additional information the four rates $R_{m}^{(186)}, R_{m}^{(189)}, R_{q}^{(186)}$, and $R_{q}^{(189)}$ were determined from the combined ${ }^{186}$ Ir and ${ }^{189}$ Ir relaxation data.

Whereas the magnetic relaxation can be characterized by a single constant, $R_{m}$, one has to specify two constants for the quadrupolar relaxation: $R_{q}^{(1)}$ and $R_{q}^{(2)}$, the contributions to $R_{q}$ that arise from $\Delta I_{z}= \pm 1$ and $\Delta I_{z}= \pm 2$ transitions, respectively [4]. The relaxation of ${ }^{189} \mathrm{Ir}$ was measured at $B_{\text {ext }}=0.2 \mathrm{~T}$ by exciting either all three subresonances of the quadrupolar split NMR spectrum or only the $\nu_{1}$ subresonance. From a comparison of this data $R_{q}^{(1)} / R_{q}^{(2)}=0.17(9)$ could be deduced. This uncertainty in $R_{q}^{(1)} / R_{q}^{(2)}$ is the main contribution to the error of $R_{q}$.
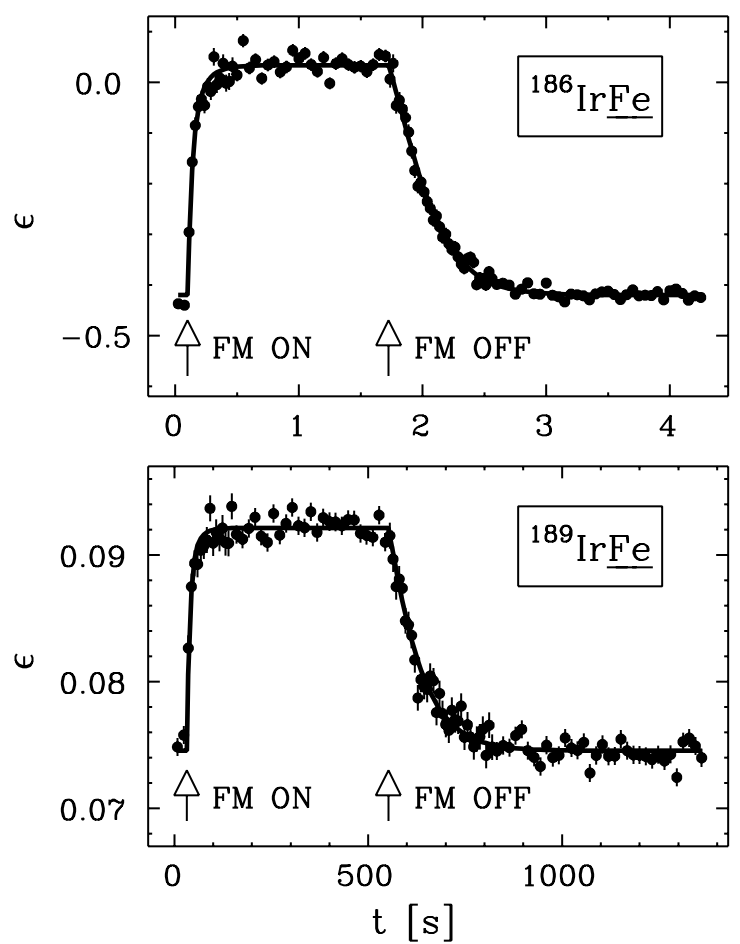

FIG. 1. NMR-ON time spectrum ( $\gamma$ anisotropy $\epsilon$ versus time during a FM off-on-off cycle) for ${ }^{186} \mathrm{Ir}$ and ${ }^{189} \mathrm{Ir}$. $B_{\text {ext }}=0.2 \mathrm{~T}$. The arrows mark the times at which the FM was switched on and off. 
It is also known that, due to the hyperfine anomaly, the Fermi-contact interaction in general does not scale exactly with $g$. This may have consequences for the scaling of $R_{m}$. The hyperfine anomaly can be estimated moderately well, but the extent to which the Fermi-contact interaction participates in the nuclear spin-lattice relaxation is a priori not known. Therefore, we give the final results for several extreme cases: for example, for a scaling of $R_{m}$ with $g^{2}$ (corresponding to a pure noncontact interaction), or for a scaling with $\nu_{m}^{2}$ (corresponding to an almost pure contact interaction).

To calculate $R_{m}^{(186)} / R_{m}^{(189)}$ and $R_{q}^{(186)} / R_{q}^{(189)}$ we used $\nu_{m}^{(189)} / \nu_{m}^{(186)}=0.12900(4), g^{(189)} / g^{(186)}=0.1174(19)$, and $Q^{(186)} / Q^{(189)}=2.902(11) . R_{m} / g^{2}$ and $R_{q} / N_{q}$ were deduced using $g^{(186)}=0.773(12)$ and $Q^{(189)}=0.878(10) \mathrm{b}$. The quadrupole moments were taken from Ref. [18]. The zero field magnetic resonance frequencies in $\mathrm{Fe}$ were measured in this work: $\nu_{m}^{(186)}=794.68(20) \mathrm{MHz}$, $\nu_{m}^{(189)}=102.512(17) \mathrm{MHz}$. The $g$ factors were derived using $\nu_{m}^{(193)}=119.00(5) \mathrm{MHz}[20], g^{(193)}=0.1061(4)$ [21], and estimating the hyperfine anomaly with the help of the Moskowitz-Lombardi rule [22] and the ratio of the contact to the total hyperfine field [23].

To check the reproducibility of our results the relaxation of ${ }^{186} \mathrm{Ir}$ was measured at $B_{\text {ext }}=0.1$ and $0.5 \mathrm{~T}$ for five different rf power levels, varying the power by a factor of 32. As expected, the deduced Korringa constants proved to be independent of the temperature and the rf power.

About $60 \%$ to $80 \%$ of the ${ }^{189}$ Ir relaxation are due to $R_{q}$. In contrast, $R_{m}$ is responsible for more than $98 \%$ of the relaxation of ${ }^{186} \mathrm{Ir}$. This allows one to determine $R_{m}$ even if only the ${ }^{186} \mathrm{Ir}$ relaxation was measured. The measured magnetic and quadrupolar relaxation constants for $B_{\text {ext }}$ between 0.01 and $1 \mathrm{~T}$ are shown in Fig. 2. In addition, $R_{m}$ was also determined at $2 \mathrm{~T}$.

The form and magnitude of the field dependence of $R_{m}$ are similar to many previous relaxation experiments on polycrystalline $\mathrm{Fe}$ samples. One difference is that the relaxation rates are constant up to $B_{\text {ext }} \leq 0.27 \mathrm{~T}$ due to the screening of $B_{\text {ext }}$ by the demagnetization field. We take here the relaxation rate at $2 \mathrm{~T}$ as the high field limit. $R_{q}$ was extrapolated to $2 \mathrm{~T}$ assuming that the form of the magnetic field dependence is the same for $R_{m}$ and $R_{q}$. Table I summarizes the final results for $R_{m}$ and $R_{q}$ at zero field and at $2 \mathrm{~T}$. The high field limit for $R_{m}$ is in moderate agreement with previous results on Ir in Fe and is about 4 times larger than predicted by the ab initio calculations [6].

The orbital relaxation was deduced from the following relation $[2,4]$ :

$$
\left(R_{o} / g^{2}\right) \leq 7.70\left(R_{q} / N_{q}\right) .
$$

This relation is valid for cubic symmetry and $d$ electrons, which dominate the orbital relaxation for the heavy $5 d$ impurities [24]. The close relationship between $R_{o}$ and $R_{q}$ arises from the fact that in both cases the nuclear spin in-

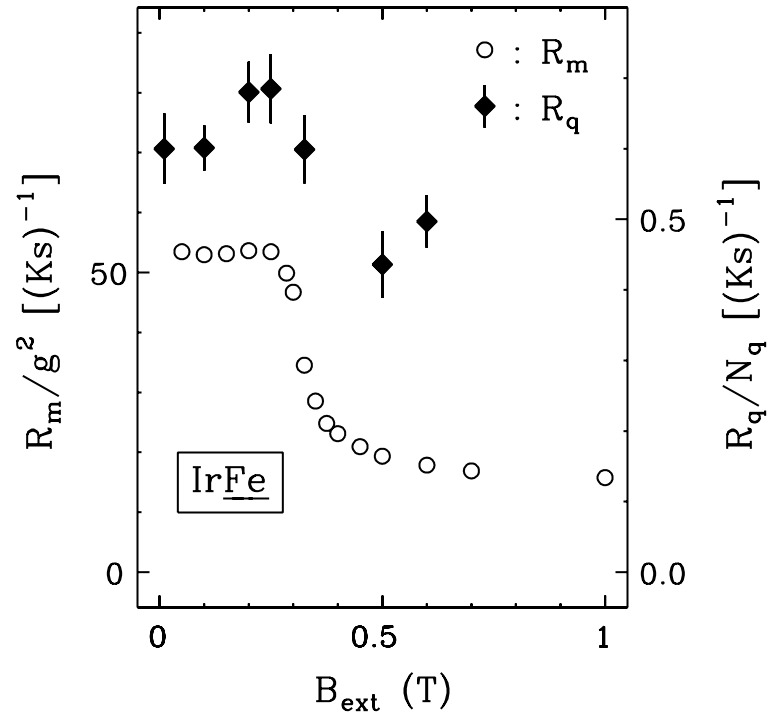

FIG. 2. The reduced magnetic and electric quadrupolar relaxation rates of $\mathrm{Ir}$ in $\mathrm{Fe}$ as a function of $B_{\text {ext }}$ applied along [100]. Because of the choice of the ordinate scales, the relative magnitude of the rates in the figure reflects the situation for ${ }^{189} \mathrm{Ir}$. The $R_{q}$ data were obtained assuming a $\nu_{m}^{2}$ scaling of $R_{m}$ and $R_{q}^{(1)} / R_{q}^{(2)}=0.17$.

teracts with the electron orbital moment and the radial part of the interaction is given by a $r^{-3}$ dependence. Table I lists the deduced $R_{o}$ 's for zero field and $2 \mathrm{~T}$. The orbital relaxation rate from the $a b$ initio calculations of Ref. [24], $R_{o}^{(t h)}$, is also listed.

A recent fully relativistic $a b$ initio calculation of $R_{q}$ (and $R_{o}$ ) for the $5 d$ impurities in $\mathrm{Fe}$ is, in principle, also available [13]. However, the results cannot, in our opinion, be correct, since the ratio $\left(R_{o} / g^{2}\right) /\left(R_{q} / N_{q}\right)$ comes out much larger (up to a factor of 30) than the factor 7.7 in Eq. (3), and the latter relation has been confirmed by previous fully relativistic calculations $[11,12]$.

The relaxation mechanism in $\mathrm{Fe}$ was discussed so far controversially: According to the $a b$ initio calculations the orbital relaxation is dominant for transition metal impurities. These calculations are believed to reproduce at least the trend of the systematics [6]. On the other hand, it was repeatedly suggested that direct and indirect spin wave mechanisms play a decisive role $[6,9,25]$. This would imply primarily spin excitations, that is, nonorbital relaxation. However, both the $a b$ initio calculations and the proposed spin wave mechanisms fail to explain the magnitude and the field dependence of the relaxation rates.

The decomposition of the relaxation into an orbital and a nonorbital part now reveals the following: (i) Experiment and $a b$ initio calculations are in reasonable agreement for the orbital part of the relaxation, at least at high fields. (ii) There is a significant field dependence of $R_{q}$, but it is at least 5 times smaller than that of $R_{m}$. We propose that this is a secondary effect, invoked perhaps via the spinorbit coupling. (iii) The unexpectedly large magnetic relaxation rates and the magnetic field dependence are 
TABLE I. Magnetic, electric quadrupolar, and orbital part of the nuclear spin-lattice relaxation of Ir in Fe. All relaxation rates are in $(\mathrm{Ks})^{-1}$.

\begin{tabular}{lccc}
\hline \hline & $B_{\text {ext }}=0$ & $B_{\text {ext }}=2 \mathrm{~T}$ & $R(0) / R(2 \mathrm{~T})$ \\
\hline$R_{m} / g^{2}$ & $53.4(17)$ & $13.81(69)$ & $3.87(15)$ \\
$R_{q} / N_{q}$ & $0.63(12)^{\mathrm{a}} \ldots 0.79(18)^{\mathrm{b}}$ & $0.45(12)^{\mathrm{a}} \ldots 0.51(15)^{\mathrm{b}}$ & $1.30(15)^{\mathrm{c}} \ldots 1.60(15)^{\mathrm{d}}$ \\
$R_{o} / g^{2}$ & $\leq 4.8(9)^{\mathrm{a}} \ldots 6.0(13)^{\mathrm{b}}$ & $\leq 3.5(9)^{\mathrm{a}} \ldots 3.9(11)^{\mathrm{b}}$ & - \\
$R_{o}^{(t h)} / g^{2}$ & $3.3^{\mathrm{e}}$ & $3.3^{\mathrm{e}}$ & 1.0 \\
\hline \hline
\end{tabular}

abtained assuming $R_{m} \propto \nu_{m}^{2}$.

${ }^{\mathrm{b}}$ Obtained assuming $R_{m} \propto g^{2}$.

cObtained assuming $R_{m}(2 \mathrm{~T}) \propto g^{2}$ and $\left[R_{m}-R_{m}(2 \mathrm{~T})\right] \propto \nu_{m}^{2}$.

${ }^{\mathrm{d}}$ Obtained assuming $R_{m}(2 \mathrm{~T}) \propto \nu_{m}^{2}$ and $\left[R_{m}-R_{m}(2 \mathrm{~T})\right] \propto g^{2}$.

${ }^{\mathrm{e}}$ Calculated $R_{o}$ from Ref. [24].

mainly due to a nonorbital relaxation mechanism. Thus, the $a b$ initio calculations themselves seem to be correct but obviously an important mechanism is missing in these calculations. The nonorbital nature of this mechanism will be important information for the further investigation of this subject. Furthermore, $R_{q}$ rather than $R_{m}$ appears to be in $\mathrm{Fe}$ the appropriate quantity used to investigate the local band structure.

To summarize, we have determined for the first time in a transition metal the electric quadrupolar part of the conduction electron contribution to the nuclear spin-lattice relaxation. This part gives information on the nature of the relaxation process. This was demonstrated for an unresolved problem in the nuclear spin-lattice relaxation of the $5 d$ impurities in Fe: Whereas the ab initio calculations predict that the orbital part dominates the relaxation in these systems, our measurements show that the bulk of the magnetic relaxation and its magnetic field dependence are of nonorbital origin.

We appreciate very much the effort which was put by the ISOLDE and Orsay groups into the development of the liquid $\mathrm{Pb}$ target. We also thank $\mathrm{E}$. Smolic for experimental help.

[1] J. Korringa, Physica (Amsterdam) 16, 601 (1950).

[2] Y. Obata, J. Phys. Soc. Jpn. 18, 1020 (1963).

[3] Y. Yafet and V. Jaccarino, Phys. Rev. 133, 1630 (1964).

[4] Y. Obata, J. Phys. Soc. Jpn. 19, 2348 (1964).

[5] T. Asada, K. Terakura, and T. Jarlborg, J. Phys. F 11, 1847 (1981).
[6] T. Funk, E. Beck, W. D. Brewer, C. Bobek, and E. Klein, J. Magn. Magn. Mater. 195, 406 (1999).

[7] T. Asada and K. Terakura, J. Phys. F 12, 1387 (1982).

[8] M. Kontani, T. Hioki, and Y. Masuda, J. Phys. Soc. Jpn. 32, 416 (1972).

[9] H. D. Rüter, W. Haaks, E. W. Duczynski, E. Gerdau, D. Visser, and L. Niesen, Hyperfine Interact. 9, 385 (1981).

[10] T. Funk, E. Klein, and W. D. Brewer, Phys. Lett. A 248, 457 (1998).

[11] R. Markendorf and C. Schober, J. Phys. Condens. Matter 7, 4561 (1995).

[12] R. Markendorf, C. Schober, and W. John, J. Phys. Condens. Matter 6, 3965 (1994).

[13] I. Cabria, M. Deng, and H. Ebert, Phys. Rev. B 62, 14287 (2000).

[14] A. Narath and D. W. Alderman, Phys. Rev. 143, 328 (1966).

[15] P. Raghavan, At. Data Nucl. Data Tables 42, 189 (1989).

[16] V. R. Green and N. J. Stone, Hyperfine Interact. 30, 355 (1986).

[17] G. Seewald, E. Hagn, E. Zech, D. Forkel-Wirth, A. Burchard, and ISOLDE Collaboration, Phys. Rev. Lett. 78, 1795 (1997).

[18] G. Seewald, E. Hagn, B. Hinfurtner, E. Zech, D. ForkelWirth, R. Eder, and ISOLDE Collaboration, Phys. Rev. Lett. 77, 5016 (1996).

[19] F. Bacon, J. A. Barclay, W. D. Brewer, D. A. Shirley, and J. E. Templeton, Phys. Rev. B 5, 2397 (1972).

[20] M. Aiga and J. Itoh, J. Phys. Soc. Jpn. 37, 967 (1974).

[21] A. Narath, Phys. Rev. 165, 506 (1968); 175, 696 (1968).

[22] P. A. Moskowitz, Phys. Lett. 118B, 29 (1982).

[23] F. E. Wagner, Hyperfine Interact. 13, 149 (1983).

[24] H. Akai, Hyperfine Interact. 43, 255 (1988).

[25] G. Seewald, E. Hagn, and E. Zech, Phys. Rev. Lett. 78, 5002 (1997). 